\title{
Recurrent Adrenal Cortex Carcinoma
}

National Cancer Institute

\section{Source}

National Cancer Institute. Recurrent Adrenal Cortex Carcinoma. NCI Thesaurus. Code C6417.

The reemergence of adrenal cortex carcinoma after a period of remission. 